\title{
FTMIR-PLS as a promising method for rapid detection of adulteration by waste whey in raw milk
}

\author{
Simone Melo Vieira ${ }^{1}$ - Letícia Maria de Souza ${ }^{2}$ (D) \\ Adriana Silva França ${ }^{1}$ - Leandro Soares Oliveira ${ }^{1}$. \\ Waldomiro Borges Neto ${ }^{2}$
}

Received: 3 February 2015 / Revised: 8 July 2015 / Accepted: 16 July 2015 /

Published online: 18 August 2015

(C) INRA and Springer-Verlag France 2015

\begin{abstract}
In this paper, we propose a feasible, sample preparation free and fast validated methodology for the detection and quantitative analysis of Minas Frescal cheese waste whey as an adulterant in raw milk using mid-infrared spectroscopy with Fourier transform (FTMIR) along with the chemometric technique of partial least squares (PLS). The PLS model was built in accordance with Brazilian and international guidelines and was analytically validated through the estimate of figures of merit parameters, in accordance with ASTM E1655-05 standard. This model showing an effective and feasible method for quality control of raw milk can be adopted for the quality control by regulatory agencies, as shown by the satisfactory results obtained for all estimated figures of merit with no systematic errors and low errors, with $R=0.99$.
\end{abstract}

Keywords Quality control · Adulteration of milk · Infrared spectroscopy·PLS . Analytical validation

Letícia Maria de Souza

leticiam_souza@yahoo.com.br; leticiams@mestrado.ufu.br

Simone Melo Vieira

simone@iftm.edu.br

Adriana Silva França

adriana@demec.ufmg.br

Leandro Soares Oliveira

leandro@demec.ufmg.br

Waldomiro Borges Neto

wbn@iqufu.ufu.br

1 Faculty of Pharmacy, Federal University of Minas Gerais, Antônio Carlos Avenue, 6627, 31270-901 Belo Horizonte, Minas Gerais, Brazil

2 Institute of Chemistry, Federal University of Uberlândia, Santa Mônica Campus, 38408-100 Uberlândia, Minas Gerais, Brazil 


\section{Introduction}

Cow's milk is an important component of the human diet because of its high nutritional value. Its nutritionally balanced composition makes it one of the most complete food available (Pereira 2014 Michalski and Januel 2006). However, the dairy market faces problems related to quality and safety of raw milk (RM) because of adulteration by adding substances with lower economic and nutritional value often occurs. Recently published studies indicate that milk is one of the most common foods involved in fraud occurrences (Aquino et al. 2014 Carvalho et al. 2007).

Minas Frescal cheese waste whey (MFCW) is a waste from Minas Frescal cheese production whose disposal is an issue of increasing importance (Carvalho et al. 2013). It is frequently used for performing this type of adulteration, since a large volume of this effluent is generated (Moore et al. 2012). The detection of this fraud is still difficult to be performed by the supervisory organizations due to similar physicochemical characteristics between MFCW and RM.

The analytical methods commonly used to detect this sort of adulteration correspond to detection of sialic acid, detection of glycomacropeptide (GMP), electrophoretic methods, immunological assays, and chromatographic techniques (Carvalho et al. 2007). These traditional analyses require a large volume of sample, are time-consuming, and demand the use of expensive and toxic chemical reagents which produce waste that can compromise the environment. Thus, it is necessary to develop simpler and faster techniques that do not generate chemical waste to be discarded into the environment and are able to accurately detect the presence of whey in milk. In this context, Fourier transform infrared spectroscopy (FTIR) has been used as an analytical method providing fast and satisfactory results and requiring only a small amount of sample without previous preparation.

Analytical validation is a requirement of ISO Guide standard (NBR ISO/IEC 17025: 2005) so that the methodology can be considered robust and reliable (Valderrama et al. 2007), and it is a fundamental condition for the PLS model that can be used by the dairy industry to investigate the quality of milk (DQOCGRE-008 2003). The validation of multivariate calibration models is obtained through the calculation of accuracy, selectivity, sensitivity, analytical sensitivity, linearity, the standard deviation, and the test of systematic errors; such parameters are known as "figures of merit" (Souza et al. 2014). Notably, a validated methodology involving detection of MFCW in RM had not been previously proposed.

In this work, we have focused on the development and validation of a PLS model using FT-MIR data applied to detection and quantification of MFCW in RM samples over the concentration range 6.0-28.0 \% (w/w), estimating the figures of merit in accordance with international and national guidelines (ASTM E1655-05 2012; Thompson et al. 2006). The range relating to adulteration was chosen according to the history of adulteration by MFCW in RM that often occurs in southeastern Brazil. 


\section{Materials and methods}

\subsection{Sampling and MIR spectroscopic data collection}

For sample preparation, we used raw milk from cattle herds from Triangulo Mineiro region in Southeast Brazil, collected over a 2-week period, and MFCW obtained from the artisanal Minas Frescal cheese production from Triangulo Mineiro region for artificial adulteration of RM. A total of $67 \mathrm{RM}$ samples were artificially adulterated with MFCW over the range 6.0-28.0\% $(w / w)$ and stored in glass vials with a final volume of $5.0 \mathrm{~mL}$. Samples were mechanically homogenized using PHOENIX AP-56/ 8821 mechanical agitator (speed approximately $250 \mathrm{rpm}$ ) and kept under refrigeration at $5.0{ }^{\circ} \mathrm{C}$ for a maximum period of $36 \mathrm{~h}$ (ISO 707|IDF 50 2008).

MIR spectra were acquired in triplicate using Perkin Elmer Spectrum. Two spectrometer equipped with a horizontal attenuated total reflectance accessory of $\mathrm{Zn} / \mathrm{Se}$ crystal (HATR-ZnSe), over the region of $4000-600 \mathrm{~cm}^{-1}$, at $4 \mathrm{~cm}^{-1}$ resolution over 16 scans, using $0.5 \mathrm{~mL}$ of each sample placed on the HATR-ZnSe crystal to obtain the spectra. The spectral baselines were corrected using the baseline method for the ranges 2600-1900 and $3750-3100 \mathrm{~cm}^{-1}$ for both data matrices. Immediately before obtaining the spectra, the samples were mechanically homogenized again, to ensure efficient mixing between RM and MFCW.

\subsection{PLS model building and analytical validation of methodology}

In order to obtain the data matrix to build the PLS model, the absorbance values of each sample in the region between 3750 and $760 \mathrm{~cm}^{-1}$ were used. The data set was mean centered and the leave-one-out method employed for cross-validation. Matlab 6.1 (Mathworks Inc.) software and PLS Toolbox 3.5 (Eigenvector Research) were used to derive the PLS models.

PLS model was constructed with a calibration set consisting of 43 samples and test set consisting of 24 samples. Prediction set was completely independent of calibration set. The number of samples for calibration and validation sets used to build the PLS model were determined according to ASTM E1655-05, which indicates that the minimum number of samples for the calibration set must be equal to $6(k+1)$ for mean-centered data (where $k$ is the number of latent variables) and the prediction set must be equal to $4 k$ (ASTM E1655-05 2012).

The number of latent variables was selected according to the percentage of variance that was observed in the $\mathbf{X}$ (absorbance) and $\mathbf{Y}$ (adulterant concentration) blocks on the joint comparison containing the plot of the root means square error of cross-validation (RMSECV).

The outlier was detected by comparing the high values of the absolute errors in the individual samples with the root means square error of calibration (RMSEC) and evaluating the high leverage values and $Q$ residuals at $95 \%$ confidence (Walczak and Massart 1998; Valderrama et al. 2009; Silva et al. 2012).

Analytical validation of PLS was performed through the calculation of parameters denominated figures of merit using net analyte signal (NAS) (Lorber et al. 1997; Ferré 
et al. 1997), in accordance with INMETRO, ISO guides, and ASTM standard for multivariate quantitative analysis of infrared data (DQO-CGRE-008 2003; ISO/IEC 17025 2005; ASTM E1655-05 2012). NAS was first proposed by Lorber et al. (1997) and has been widely discussed in the literature (Souza et al. 2014; Ferré et al. 1997) and defined as the part of the analytical signal uniquely related to the analyte of interest and orthogonal to the interferences.

The accuracy (Eq. 1) which corresponds to the degree of agreement between the reference value and the predicted value using the PLS model (Souza et al. 2014) was determined by calculating the RMSEC, RMSECV, and root means square Error of Prediction (RMSEP). The elliptical joint confidence region was held for the evaluation of systematic and random errors (Souza et al. 2014).

Linearity corresponds to the ratio between the MFCW concentration values predicted by PLS model and the real values of MFCW concentration for each sample which is calculated by Eq. 2. The fit graphs of PLS model were obtained correlating the real values with the predicted values from the prediction set and using the graph of real values versus the NAS norms (Souza et al. 2014; Silva et al. 2012; Lorber et al. 1997).

The NAS value was applied to calculation of selectivity, and this parameter was related to the determination of the amount of the instrumental signal that was used by the model for the determination of the MFCW, as shown in Eq. 3 (Souza et al. 2014; Silva et al. 2012).

Equation 4 was used to calculate the sensitivity parameter, defined as the signal fraction responsible for the increased of one unit of concentration for MFCW (property of interest in sample) (Lorber et al. 1997; Rocha et al. 2012).

Analytical sensitivity (Eq. 5) corresponded to the ratio between sensitivity and the estimated standard deviation of instrumental noise $\left(\delta_{\mathrm{x}}\right)$, and the inverse of this parameter $\left(\gamma^{-1}\right)$ expressed the minimum difference in concentration that can be discernible by this method, considering the instrumental noise as the only source of error, whatever the technique employed (Souza et al. 2014; Rocha et al. 2012; Lorber et al. 1997). $\delta_{\mathrm{x}}$ was obtained using nine replicate spectra of the RM free of addition of MFCW, following the IUPAC recommendations (Thompson et al. 2006).

The value of "bias" used to investigate the presence of systematic errors in PLS model was obtained using Eq. 6, calculated from the difference between the mean and references values, and are the components of error that are not random. The standard deviation validation value (SDV) characterizes the dispersion of predicted adulteration values by validation set and was calculated by Eq. 7 (Souza et al. 2014; Silva et al. 2012).

ASTM E1655-05 standard mentions that the investigation of systematic errors should be performed using a $t$ test (Eq. 8) for validation samples at $95 \%$ confidence and degrees of freedom equal to the number of prediction samples (ASTM Standard E1655-05 2012).

\section{Results and discussion}

The spectral profiles of the samples (Fig. 1) show many similarities in absorbance bands and little variations, and these variations in absorbance of certain regions are not directly related to variations in adulterant concentrations, requiring chemometric tools 


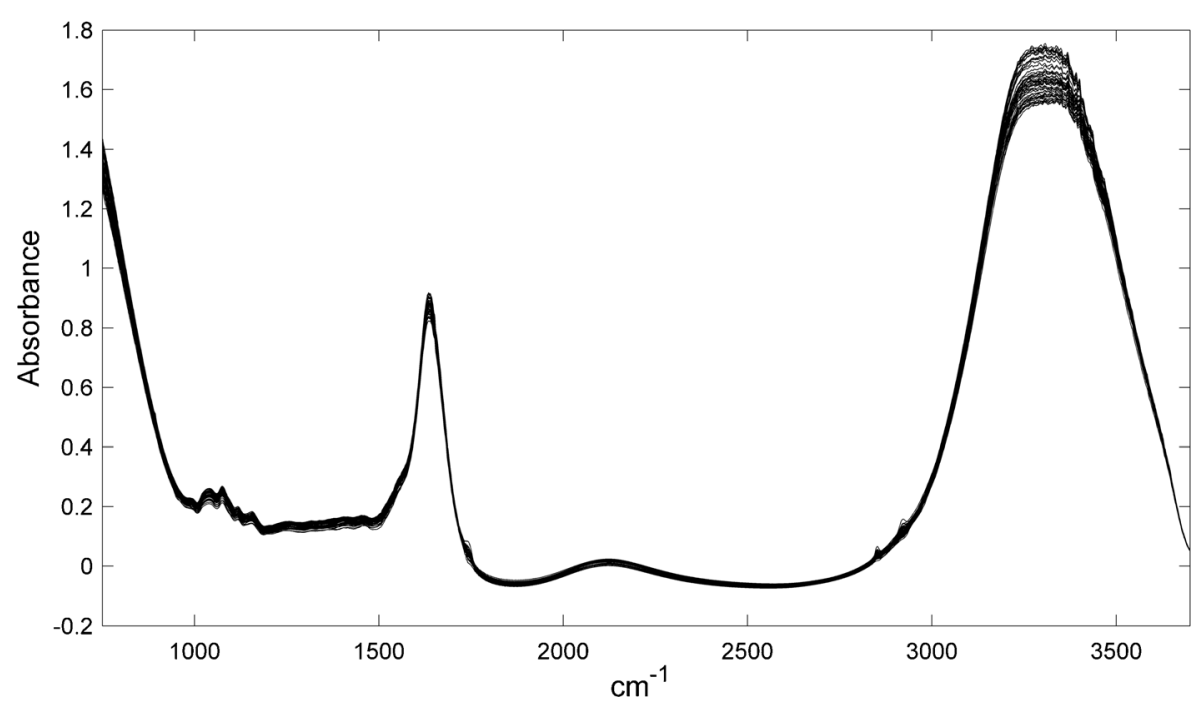

Fig. 1 MIR spectra of samples of raw milk $(R M)$ adultered with Minas Frescal cheese whey $(M F C W)$ adulteration levels from 6.0 to $28.0 \%(w / w)$

that allow the quantification of MFCW in RM. However, the slight increase in absorption bands were observed at $1620 \mathrm{~cm}^{-1}$ which can be correlated to absorption of $\beta$ structures of the proteins present in the $\beta$-lactoglobulins which are dispersed in MFCW.

Table 1 Figures of merit parameters calculated for the PLS model prediction Minas Frescal cheese whey $(M F C W)$ in raw milk $(R M)$ samples

\begin{tabular}{|c|c|c|c|c|}
\hline Figures of merit & Equations & & Parameter & Value \\
\hline Accuracy & \multicolumn{2}{|c|}{$\mathrm{RMSE}=\sqrt{\frac{\sum_{\mathrm{i}=1}^{\mathrm{n}}\left(\mathrm{y}_{\mathrm{i}}-\hat{\mathrm{y}}_{\mathrm{i}}\right)^{2}}{\mathrm{n}}}($ Eq. 1) } & $\begin{array}{l}\text { RMSEC }(\% w / w) \\
\operatorname{RMSECV~}(\% w / w) \\
\operatorname{RMSEP}(\% w / w)\end{array}$ & $\begin{array}{l}0.11 \\
0.16 \\
0.18\end{array}$ \\
\hline Linearity & $R=\frac{\sum_{i-1}^{n}\left|\frac{y_{i}}{y_{i}}\right|}{n}$ & (Eq. 2) & $R$ & 0.99 \\
\hline Selectivity & $S E L_{i}=\frac{\widehat{n a s_{i}}}{\left\|\boldsymbol{x}_{\mathrm{i}}\right\|}$ & (Eq. 3) & $(\%)$ & 0.01 \\
\hline Sensitivity & $\mathrm{SêN}=\frac{1}{\left\|\mathrm{~b}_{\mathrm{k}}\right\|}$ & (Eq. 4) & & 0.11 \\
\hline Analytical sensitivity & $\gamma=\frac{S \hat{e} N}{\delta \mathrm{x}}$ & (Eq. 5) & & 38.67 \\
\hline Inverse of analytical sensitivity & & & & 0.38 \\
\hline \multirow[t]{5}{*}{ Systematic error } & bias $=\frac{\sum_{i=1}^{n_{\text {val }}}\left(y_{i}-\hat{y}_{i}\right)}{n_{\text {val }}}$ & (Eq. 6) & Bias & 0.04 \\
\hline & & & Standard deviation & 0.18 \\
\hline & $S D V=\sqrt{\frac{\sum\left[\left(y_{i}-\hat{y}_{i}\right)-\text { bias }\right]^{2}}{n_{\text {val }}-1}}$ & (Eq. 7) & Degree of freedom & 24 \\
\hline & & & $\mathrm{t}_{\mathrm{calc}}$ & 1.33 \\
\hline & $t_{\text {bias }}=\frac{\mid \text { bias } \mid \sqrt{n_{\text {val }}}}{S D V}$ & (Eq. 8) & $\mathrm{t}_{\text {crit }}$ & 2.06 \\
\hline
\end{tabular}

In Eqs. 1 and $2, \hat{y}_{i}$ is the predicted value using the PLS model, $y_{i}$ is the reference value for the sample $I$, and $n$ is the number of samples. In Eq. 2, nâs $s_{i}$ is the norm of the NAS vector and $\boldsymbol{x}_{\mathrm{i}}$ is the norm of each spectrum. In Eq. 3, $\mathbf{b}$ is the vector of final regression coefficients, which can be obtained by any multivariate method 


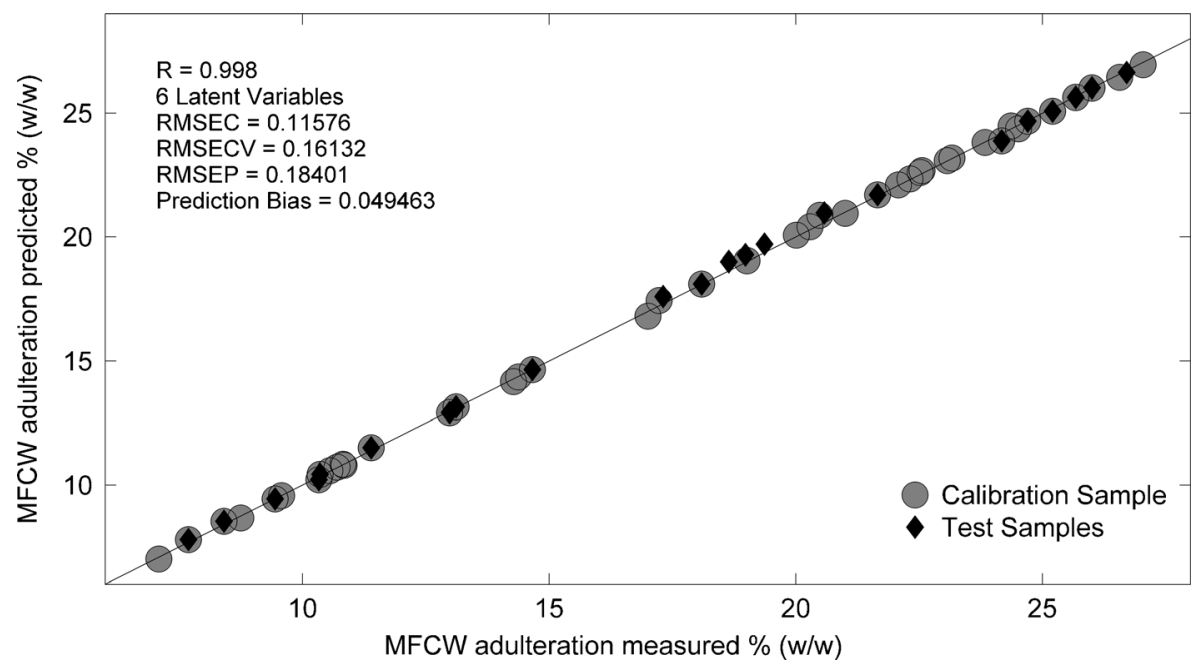

Fig. 2 PLS model fit for raw milk $(R M)$ samples adulterated by Minas Frescal cheese whey $(M F C W)$

The equations used to calculate the figures of merit and parameters estimated are shown in Table 1. The PLS model was built using six latent variables and showed a good fit between the predicted and measured values, with $R=0.998$ (Fig. 2).

The ratio between $Q$ residual and leverage values was used to verify the presence of outliers in the PLS model. We observed that none of the samples was considered an outlier in the IR region evaluated, with $95 \%$ confidence limits. In addition, no outlier was detected by high absolute errors of the individual samples upon comparing the RMSEC value for model.

The results achieved for the figures of merit have confirmed the accuracy and linearity of the model; PLS model showed low values of RMSEC, RMSECV, and

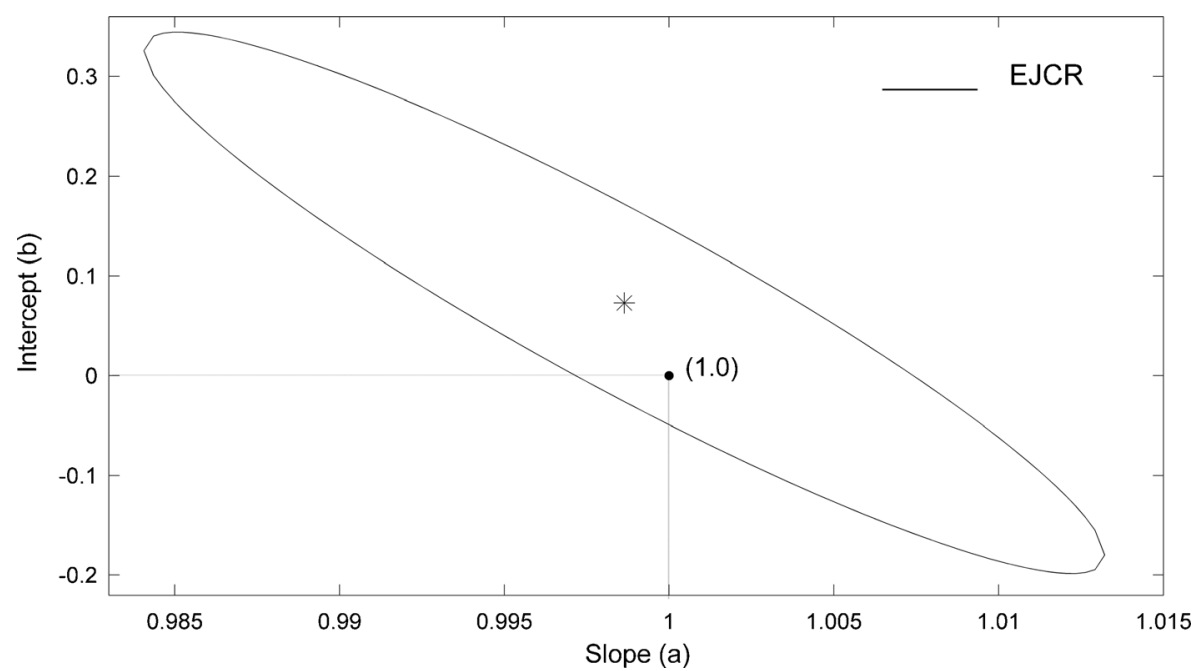

Fig. 3 The elliptical joint confidence region $(E J C R)$ for the slope and intercept of the regression of predicted concentration versus the reference values. The ideal result consisting of intercept $=0$ and slope $=1$ is shown by point, whereas the experimental result for PLS model is indicated by asterisk, with $a=0.9986$ and $b=0.0730$ 


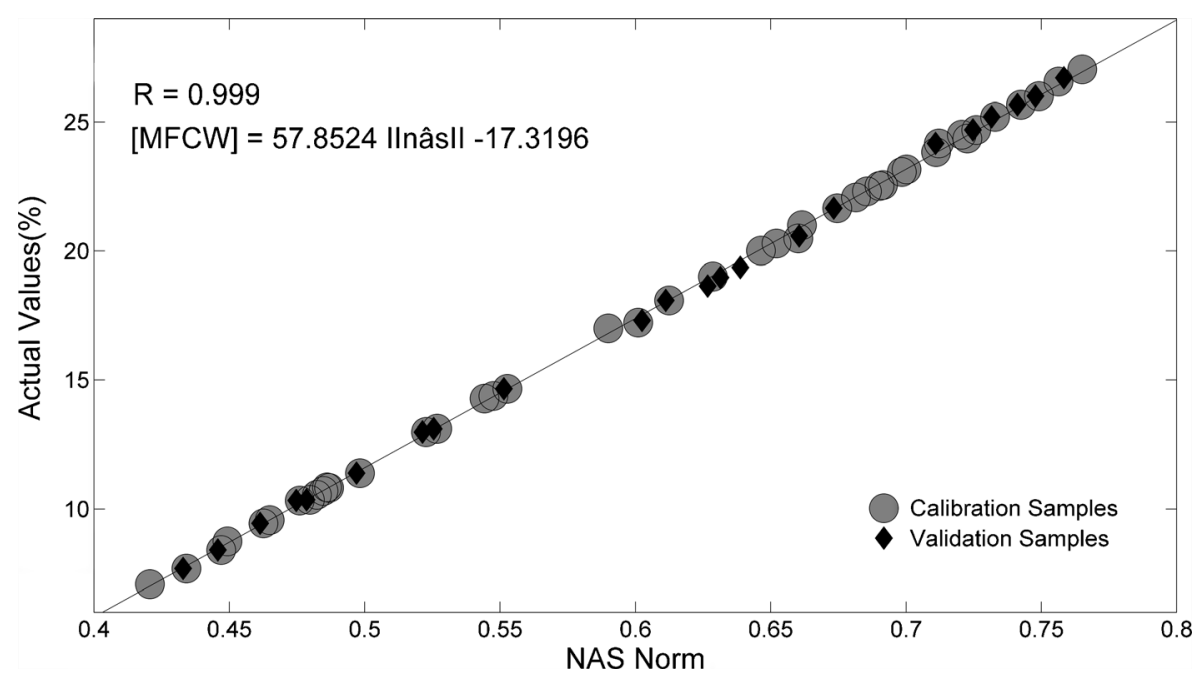

Fig. 4 Pseudo-univariate calibration curve. Plot of the norm of the NAS versus reference values for the calibration and validation samples for raw milk (RM) with Minas Frescal cheese whey (MFCW)

RMSEP and was considered acceptable and efficient for quality control of RM and detection of MFCW adulteration at range of 6.00-28.00\% (w/w).

The elliptical joint confidence region (EJCR) test was applied to the estimated intercept (b) and slope (a); these data were compared with the ideal values of $b=0$ and $a=1$. In this case, an ordinary least squares fitting of the prepared concentration values versus the predicted concentration values for model was used. Figure 3 shows the EJCR for the results of the PLS model, which noted the lack of significant difference between the values of concentration of artificial adulteration and the PLS predicted concentration values, and that there was no evidence of bias within the $95 \%$ confidence level with $b=0.0730$ and $a=0.9986$. Therefore, the PLS model was able of quantifying MFCW in RM.

Linearity parameter was evaluated using errors obtained from the PLS model, as $R$ value, as shown in Fig. 2. However, the linearity can also be expressed in relation to the NAS values; the equations in Fig. 4 are particularly useful in routine analysis, where the analyst can view the PLS model in a univariate way.

PLS model showed no systematic errors by the $t$ test which was applied to assess whether the bias included in the model is significant. In the PLS model, $t_{\text {calc }}<t_{\text {critc }}$ for the degree of freedom equal to 24 , which corresponds to the number of prediction samples.

\section{Conclusion}

This paper proposes an efficient, low-cost, fast, and sample preparation-free methodology to determine adulteration by addition of cheese waste whey in raw milk. The PLS model was built based on the FT-MIR spectroscopy of a representative set of samples of RM containing 6.0-28.0\% $(w / w)$ MFCW. The method was validated, as shown by the satisfactory results obtained for all estimated figures of merit with no systematic 
errors. Moreover, the proposed method presents other advantages, such as simplified procedure, no need for reagents or solvents, and less generation of chemical waste. Therefore, this HATR-MIR-PLS methodology demonstrates great potential as an attractive alternative for the quantification of MFCW in RM and can be adopted as an efficient method for the quality control of milk by regulatory agencies.

Acknowledgments The authors would like to acknowledge CAPES and FAPEMIG for their financial support.

Conflict of interest Authors Simone Melo Vieira, Letícia Maria de Souza, Adriana Silva França, Leandro Soares Oliveira, and Waldomiro Borges Neto declare that they have no conflict of interest.

Statement of human and animal rights This article does not contain any studies with human or animal subjects performed by any of the authors.

\section{References}

ABNT NBR ISO/IEC 17025:2005. Brazilian Association of Technical Standards (2005) General requirements for the competence of testing and calibration laboratories, Rio de Janeiro, Brazil <http://www.inmetro. gov.br>

Aquino LFMC, Silva ACO, Freitas MQ, Felicio TL, Cruz AG, Conte-Junior CA (2014) Identifying cheese whey an adulterant in milk: limited contribution of a sensometric approach. Food Res Int 62:233-237

ASTM Standard E1655-05 (2012) Standard practices for infrared multivariate quantitative analysis, ASTM International, West Conshohocken, PA $<$ www.astm.org $>$

Carvalho BMA, Carvalho LM, Ayra L, Alcântara P, Bonono RCF (2007) Methods for fraud detection in milk by adding cheese whey. Electron J Vet 8(6):1695-7504

Carvalho F, Prazeres AR, Rivas J (2013) Cheese whey wastewater: characterization and treatment. Sci Total Environ 445-446:385-396

Ferré J, Boqué R, Fernández-Band B, Larrechi MS, Rius FX (1997) Figures of merit in multivariate calibration. Determination of four pesticides in water by flow injection analysis and spectrophotometric detection. Anal Chim Acta 348:167-175

INMETRO DQO-CGRE-008 (2003) Guidelines on validation of chemical tests. National Institute of Metrology, Standardization and Industrial Quality, Brazil < www.inmetro.gov.br>

ISO 707|IDF 50:2008 (2008) Milk and milk products—guidance on sampling, Requirements for milk sampling and milk products for microbiological, chemical, physical and sensory analyzes, except for (semi) automatic sampling <www.iso.org $>$

Lorber A, Faber K, Kowalski BR (1997) Net analyte signal calculation in multivariate calibration. Anal Chem 69:1620-1626

Michalski MC, Januel C (2006) Does homogenization affect the human health properties of cow's milk? Trends Food Sci Technol 17:423-437

Moore JC, Spink J, Lipp M (2012) Development and application of a database of food ingredient fraud and economically motivated adulteration from 1980 to 2010. J Food Sci 77:118-126

Pereira PC (2014) Milk nutritional composition and its role in human health. Nutrition 30(6):619-627

Rocha WF, Nogueira R, Vaz BG (2012) Validation of model of multivariate calibration: an application to the determination of biodiesel blend levels in diesel by near-infrared spectroscopy. J Chemom 26:456-461

Silva MAM, Ferreira MH, Braga JWB, Sena MM (2012) Development and analytical validation of a multivariate calibration method for determination of amoxicillin in suspension formulations by near infrared spectroscopy. Talanta 89:342-51

Souza LM, Mitsutake H, Gontijo LC, Borges Neto W (2014) Quantification of residual automotive lubricant oil as an adulterant in Brazilian S-10 diesel using MIR spectroscopy and PLS. Fuel 130:257-262

Thompson M, Ellison SLR, Wood R (2006) The international harmonized protocol for the proficiency testing of analytical chemistry laboratories (IUPAC technical report). Pure Appl Chem 78(1):145-196

Valderrama P, Braga JW, Poppi RJ (2007) Variable selection, outlier detection, and figures of merit estimation in a partial least-squares regression multivariate calibration model. A case study for the determination of 
quality parameters in the alcohol industry by near-infrared spectroscopy. J Agric Food Chem 55:83318338

Valderrama P, Braga JWB, Poppi RJ (2009) State of the art of figures of merit in multivariate calibration. Quim Nov. 32(5):1278-1287

Walczak B, Massart DL (1998) Multiple outlier detection revisited. Chemom Intell Lab Syst 41:1-15 